\title{
PRÁCTICAS PARENTALES E INTELIGENCIA EMOCIONAL EN ESTUDIANTES DE SECUNDARIA. UN ESTUDIO CORRELACIONAL
}

\author{
José Fernando Juarez Flores \\ Benemérita Universidad Autónoma de Puebla. \\ Facultad de Psicología \\ psi.josefjflores@gmail.com \\ Rocío Fragoso Luzuriaga \\ Benemérita Universidad Autónoma de Puebla \\ Facultad de Psicología \\ psicrociofras@gmail.com
}

Fecha de Recepción: 9 Agosto 2019

Fecha de Admisión: 25 Septiembre 2019

\section{RESUMEN}

La familia tiene una influencia determinante en el desarrollo de habilidades socioemocionales, como la Inteligencia Emocional (IE), en niños y adolescentes. Sin embargo, actualmente existen pocos estudios empíricos que ayuden a profundizar en la temática. Por lo anterior, la presente investigación tuvo como objetivo determinar las correlaciones existentes entre la IE y las Practicas Parentales percibidas por estudiantes de $2^{\circ}$ año de la Secundaria Técnica Número 96 de la ciudad de Puebla. Para lograr dicho propósito se aplicó el TMMS-24 junto con la Escala de Prácticas Parentales para Adolescentes de Andrade Palos y Betancourt Ocampo (2008) a 196 jóvenes entre los 13 y 15 años de edad. Posteriormente, al analizar los resultados de ambos instrumentos, se encontró que las prácticas parentales de autonomía, comunicación y control conductual se correlacionan positivamente con la IE en tanto que las prácticas parentales de control psicológico se correlacionan negativamente con la IE.

Palabras clave: padres; inteligencia; emoción; adolescentes

\section{ABSTRACT}

Parental practices and emotional intelligence in junior high students. A correlational study. The family is a determinant factor on the development of socio-emotional skills, such as Emotional Intelligence (EI), in children and adolescents. However, there are few empirical research about the topic. Therefore the aim of this article is to analyze the correlations between El and Parental Practices in students enrolled at Junior High School Number 96 in Puebla city. To fulfill this purpose, two instruments were applied: The TMMS-24 and Parental Practices Scale for Adolescents designed by Andrade \& Betancourt (2008) on 196 subjects between 13 and 15 years old. The results indicate 


\section{PRÁCTICAS PARENTALES E INTELIGENCIA EMOCIONAL EN ESTUDIANTES DE SECUNDARIA. UN ESTUDIO CORRELACIONAL}

that parental practices of autonomy, communication and behavioral control correlate positively with El while the parental practices of psychological control are negatively correlated with El.

Keywords: parents; intelligence; emotion; adolescence

\section{INTRODUCCIÓN}

El sistema familiar ha sufrido numerosas transformaciones a través del tiempo relacionadas con su tamaño, interacciones, normas, roles y formas de comunicación como parte de su adaptación a entornos sociales cada vez más complejos. Sin embargo, su objetivo principal, que es asegurar el óptimo desarrollo físico y psicológico de sus miembros, no ha cambiado en la época contemporánea (Rodrigo López \& Palacios, 1998; Vázquez Rúa, 2005).

Para cumplir con ese propósito cada uno de los subsistemas que la integran ha de cumplir una serie de funciones que los convierte en catalizadores del fomento de habilidades socioemocionales que determinan la manera en que un individuo se integrará al ambiente que le rodea (Cuervo Martínez, 2009; Bilimler Dergisi, 2006). Por ejemplo: el subsistema de los hermanos, brinda compañía, solidaridad y afecto dentro del binomio afecto-rivalidad y entrena en la interacción con otros individuos, pero cuando éste no existe, el subsistema padres e hijos aporta estos elementos y al mismo tiempo provee seguridad y confianza a través de la generación de vínculos emocionales; el subsistema de la pareja, base del sistema familiar, no sólo brinda espacios de comunicación, intimidad y expresión emocional que contribuyen al crecimiento de sus miembros alentando el logro de proyectos personales, también, al iniciarse la paternidad, crea un proyecto vital al que se suman tareas de crianza y socialización que han de conquistarse empleando ciertos recursos y habilidades entre los que destacan las prácticas parentales y el desarrollo de la IE (Rodrigo López \& Palacios, 1998; Espinal, Gimeno y González, 2004), conceptos que se desarrollan enseguida:

Las prácticas parentales, también llamadas prácticas de crianza, estrategias de socialización 0 estilos educativos, son aquellas que crean el ambiente psicológico donde se desenvolverán los hijos a lo largo de las diferentes etapas del desarrollo al tiempo que transmiten un conjunto de valores y normas que facilitan la incorporación al grupo social. Estas se fundan tanto en la edad, el sexo, el orden de nacimiento y la personalidad de los menores como en la experiencia de los padres siendo hijos, el nivel educativo de los progenitores así como sus ideas sobre el proceso evolutivo y las expectativas sobre la crianza (Cuervo Martínez, 2010; Darling \& Steinberg, 1993; Ramírez, 2005).

El estudio de las prácticas parentales ha demostrado que aquellas basadas en el dialogo y la concesión de autonomía tienen mejores efectos ya que se relacionan con competencias como la empatía, autocontrol, autoestima, sociabilidad y menor impulsividad. Además, brindan confort y apego seguro en la relación padres e hijos al permitir el intercambio verbal una vez que se presentan diferencias (Baumrind, 1966). En contraste, el empleo de prácticas negativas produce bajos niveles de comunicación (Méndez Sánchez, Andrade Palos \& Peñaloza Gómez, 2013), dificultades en el aprendizaje y la atención (Malander, 2016), síntomas depresivos y ansiosos (Meier \& Oros, 2012), competencias socioemocionales deficientes, problemas conductuales y dificultades en la vida diaria (Palacios Delgado \& Andrade Palos, 2008).

Por otro lado, la IE es definida por Mayer y Salovey (1997) como la habilidad para percibir las emociones, acceder a ellas, utilizarlas para asistir al pensamiento, comprenderlas y regularas de manera reflexiva con el propósito de promover el crecimiento personal. Las investigaciones revelan que óptimos niveles de la habilidad se relacionan con un buen funcionamiento del sistema familiar debido a que incide en la comunicación, la libre expresión y las formas funcionales de organización de los miembros que lo integran (Gil Olarte, Guil Bozal, Serrano Díaz y Larrán Escandón, 2014). Sus beneficios se extienden a áreas como la salud mental ya que tiene efectos sobre la autoestima y 
autorregulación disminuyendo las posibilidades de desarrollar alteraciones de la personalidad, problemas de conducta 0 depresión (Frías Armenta, Fraijo Sing \& Tapia Fonllem, 2012); también se relaciona con rasgos positivos de la personalidad y aspectos generales de la inteligencia (Mestre Navas, Guil Bozal \& Mestre Romero, 2005); adecuado ajuste psicológico (Cobos Sánchez, Flujas Contreras \& Gómez Becerra, 2017), buen rendimiento académico, bienestar psicológico y buenas relaciones interpersonales (Ferragut \& Fierro, 2012; Fernández Berrocal \& Ruíz Aranda, 2008).

El fomento de la IE y el perfeccionamiento de prácticas parentales efectivas deben buscarse durante todo proceso de crianza, aunque, resultan particularmente importantes en la adolescencia pues impactan positivamente en la manera de hacer frente a los factores de riesgo presentes en otros sistemas, como la escuela 0 el grupo de amigos. En adición, disminuyen la vulnerabilidad ante las crisis que supone la etapa, sin embargo, pese a los beneficios que parecen compartir ambos constructos, su estudio se ha llevado a cabo por separado (Bathia, 2012; Papalia, Wendkos Olds \& Duskin Fieldman, 2009).

Es cierto que las prácticas parentales se han relacionado con algunos aspectos emocionales pero no se ha explorado cómo impactan sobre el desarrollo de las habilidades que integran la IE. Aunado a ello, en las investigaciones, los estilos de crianza siempre han sido abordados desde la perspectiva adulta dejando de lado que la relación padres-hijos no es lineal, lo cual supone que estos últimos influyen en la forma cómo se les educa. Esto hace que deba considerarse la manera en que los niños y jóvenes perciben las prácticas parentales pues de no hacerlo se corre el riesgo de pensar ser un padre abierto al dialogo, cariñoso y responsable mientras que los hijos perciben todo lo contrario (Capano Bosch, Del Luján González y Massonier, 2016; Frías Armenta et al., 2012; Cuervo Martínez, 2010).

Por todo lo anterior surgió la siguiente pregunta de investigación: ¿Existe correlación entre las diferentes prácticas parentales percibidas (comunicación, autonomía, imposición, control conductual y psicológico) y las habilidades que integran la IE en estudiantes de segundo grado de secundaria?

Congruente con el cuestionamiento anterior surgen las siguientes hipótesis de investigación:

$\mathrm{H}_{\mathrm{j}}$ : Existe una correlación entre las diferentes prácticas parentales percibidas y las habilidades de la IE en estudiantes de segundo grado de secundaria

$\mathrm{H}_{0}$ : No existe una correlación entre las diferentes prácticas parentales percibidas y las habilidades de la IE en estudiantes de segundo grado de secundaria

Por añadidura el objetivo general de este trabajo es determinar la correlación que existente entre las prácticas parentales percibidas por los adolescentes y las habilidades de la IE que poseen.

Así pues, el artículo está organizado de la siguiente manera: en la Introducción, se abordan los elementos generales que rigen la investigación, así como su fundamentación teórica; en la metodología se expone el tipo de estudio, así como una descripción de los sujetos que participaron y de los instrumentos empleados para la recolección de datos. Posteriormente, en el apartado de los resultados se expone la información obtenida de las variables estudiadas, estilos parentales e IE, así como las correlaciones encontradas y, finalmente, se presentarán las conclusiones y la discusión del análisis de la temática.

\section{Prácticas Parentales}

Si bien el auge por averiguar el modo en que ciertas conductas familiares afectan el desarrollo de los niños comenzó con los trabajos de Baumrind (1966) la autora únicamente elaboró una tipología de padres en función de las prácticas empleadas con sus hijos. Las definiciones de lo que son las prácticas parentales derivan de investigaciones posteriores y parten de las dimensiones de estu- 


\section{PRÁCTICAS PARENTALES E INTELIGENCIA EMOCIONAL EN ESTUDIANTES DE SECUNDARIA. UN ESTUDIO CORRELACIONAL}

dio con las que se les relacione, así pues, Capano y Ubach (2013) parten de la tarea de crianza para definirlas como un fenómeno específico de cierta permanencia y estabilidad en el tiempo que permite a los padres educar y que según las condiciones y circunstancias de cada familia pueden modificarse, definición con la que coincide Ramírez (2005). Barudy y Dantagnan (2005), sin embargo, interesados en el buen trato a la infancia las explican como capacidades que los padres deben desarrollar para asegurar el sano desarrollo de sus hijos al permitirles satisfacer sus necesidades y junto con la capacidad de apego, empatía y construcción de redes sociales, así como la habilidad de adaptarse a las diferentes etapas de sus hijos constituyen lo que denominan competencias parentales.

A su vez, Cuervo Martínez (2010) parte de entender a la familia como una entidad que debe enseñar valores y normas que permitan a los menores integrarse a lo social, por lo que describe estas practicas como la manera en que se les orienta en tal fin, esta definición es cercana a la de Darling y Steinberg (1993) en el sentido de que ambas consideran la socialización pero los dominios de interés no son los valores sino el logro académico, la independencia lograda.

Del mismo modo, las clasificaciones de estas prácticas están determinadas en función de las dimensiones consideradas por los autores, Barudy y Dantagnan (2005), por ejemplo, clasifican las prácticas en sanas, competentes y bientratantes o disfuncionales incompetentes y maltratantes según sea la calidad del ambiente cognitivo y social, así como el apego que los padres promuevan con sus hijos y agregan que la parentalidad disfuncional puede ser autoritaria como resultado del temor de los niños ante sus padres, o bien permisiva al no prestar atención de las necesidades infantiles, pero según estas variables los padres pueden ser represivos-autoritarios, permisivos-indulgentes o permisivos-negligentes.

Por su parte, Nardone, Giannoti y Rocchi, (2003) analizando la manera en que se comporta la familia de los adolescentes mencionan que los padres pueden ser hiperprotectores, limitando las experiencias de sus hijos; democráticos-permisivos, desdibujando las jerarquías; sacrificantes, afectando la autorrealización de sus hijos; intermitentes, sin pautas claras de conducta y normas cambiantes; delegantes, quienes siempre ven a sus hijos como niños indefensos o bien autoritarios en el que la obediencia es la obligación.

En cuanto al estudio de Baumrind (1967) ubicado en los modelos de construcción conjunta, modelo de investigación que permite hacer correlaciones a partir de la selección de variables de estudio que al ser evaluadas permiten una aproximación significativa al sistema estudiado (Aroca Montolío y Cánovas Leonhardt, 2012), la autora partió de la hipótesis de que el desarrollo físico, cognitivo y social de los niños está en gran parte relacionado con las prácticas de sus padres por lo que evaluó cómo las dimensiones control, comunicación e implicación afectiva impactaban las competencias de autocontrol, tendencia a evitar el acercamiento, autosuficiencia, humor subjetivo y afiliación entre iguales de los menores, según el grado observado de estas capacidades en los niños Baumrind los dividió en tres grupos. Un primer patrón estuvo formado por aquellos niños y niñas que fueran autosuficientes, curiosos, alegres y con capacidad de autocontrol y los contrastó con niñas y niños descontentos, distantes y desconfiados, mismos que conformaban un segundo patrón y un tercero en el que las y los menores mostraran bajo autocontrol y autosuficiencia y retracción ante experiencias novedosas y verificó la manera en que estas características estaban relacionadas con las dimensiones paternas consideradas encontrando que:

Los padres de hijos en el primer patrón resultaron ser los más amorosos, consistentes, conscientes y seguros al tratar a sus hijos y también respetaban las decisiones de los niños, pero demostraron una marcada habilidad para sostener sus decisiones una vez que tomaban una postura y eran directivos, aunque explicando las razones para ello. Las madres de estos niños mostraron control firme y demandaban un buen trato de sus hijos, los apoyaban más y se comunicaban de manera 
clara lo cual no ocurría con los padres cuyos hijos estaban en el patrón dos y tres. Y aunque la interacción podía llegar a ser conflictiva en ocasiones los hogares de estos niños no se caracterizaban ni por la discordia ni por la disensión.

Del mismo modo, estos padres balanceaban la calidad de sus cuidados con el control ejercido y la manera en que se comunicaban con ellos, todas estas características diferían con los padres de niños y niñas en los grupos dos y tres. Los hijos de estos padres y madres no fueron negativamente afectados por las demandas de madurez y socialización y, de hecho, parecían prosperar ante las situaciones de presión impuestas.

Los padres con hijos del segundo grupo estaban menos involucrados en la crianza de sus hijos y, además, eran menos amorosos. Su manera de ejecutar el control era firme y abierta, aunque ofrecían escaso afecto y apoyo e intentaban convencer a sus hijos sin escucharlos cuando disentían de las actitudes parentales. La madre estaba más inclinada a dar imperativos morales como razones para justificar sus acciones y su actitud era menos empática y aprobatoria y admitieron asustar a sus hijos con el fin de educarlos.

En cuanto al tercer grupo, ninguno de los padres solicitaba demasiado de sus hijos y los reforzadores empleados en la crianza eran deficientes. En contraste con los padres del patrón uno éstos estaban aún menos involucrados y usaban muestras de amor de manera manipuladora, retiraban sus afectos y los ridiculizaban en lugar de emplear argumentos razonables o incentivos para lograr conductas deseables. La diferencia más significativa entre los padres del patrón dos y los del patrón tres era que los primeros resultaron ser más controladores.

Estos resultados llevaron a Baumrind (1967) a clasificar a los padres en:

Autoritativos/democráticos: Estos dirigen racionalmente la conducta de sus hijos, orientan con preguntas y alientan el "dar y recibir" verbal por lo que comparten con ellos las razones que existen detrás de las reglas que imperan en la familia y se negocia cuando no se está de acuerdo con ellas, lo cual no evita que se aplique control firme ante situaciones divergentes en la relación padre-hijo. También hacen valer su perspectiva como adultos, pero reconocen los intereses y puntos de vista de sus hijos, al tiempo que reafirman sus cualidades y estructuran escenarios para futuras conductas. Utilizan la razón, poder y formación por régimen y reforzamiento para lograr sus objetivos y no basan sus decisiones en el consenso del grupo 0 en los deseos del niño.

Autoritarios: Estos forman, controlan y evalúan el comportamiento y actitudes de los niños de acuerdo con un conjunto de estándares conductuales rígidos e inflexibles. La virtud más apreciada es la obediencia, por ello restringen la autonomía, manteniéndolos en su lugar y asignándoles tareas que les hagan respetar el trabajo, lo que también pretende mantener el orden y las estructuras tradicionales utilizando una estrategia: quitar toda voz a sus hijos para que acepten que todo cuanto dicen sus padres es lo mejor.

Permisivos: Estos padres se comportan de manera afirmativa, no punitiva y proveyendo aceptación de los deseos, impulsos y acciones de los niños. Consultan con sus hijos e hijas las reglas que han de implementarse en la familia, además de que hace pocas demandas y asigna pocas responsabilidades, presentándose como recurso siempre dispuesto, aunque eso no significa que sea un ejemplo a seguir pues permite que los niños regulen como puedan sus propios comportamientos y actividades. Para lograr sus objetivos educativos, los padres recurren a la manipulación y a dar razones poco creíbles.

Posteriormente, este modelo sería reformulado por MacCoby y Martin partiendo de las dimensiones de estudio control o exigencia, afecto o sensibilidad, y calidez, añadiendo el estilo negligente para describir a padres que ejercen control nulo sobre sus hijos y que, al mismo tiempo, no tienen ningún grado de calidez con ellos. 


\section{PRÁCTICAS PARENTALES E INTELIGENCIA EMOCIONAL EN ESTUDIANTES DE SECUNDARIA. UN ESTUDIO CORRELACIONAL}

\section{Inteligencia Emocional}

Desde su nacimiento a manos de los científicos Salovey y Mayer (1990), la IE ha llamado la atención de numerosos autores como Goleman (2000, 2002), Cooper y Sawaf (1996), Bisquerra Alzina $(2007,2009)$ y BarOn (2006) quienes han creado su propia interpretación del concepto. No obstante, algunos de ellos no han contado con la solidez metodológica de sus creadores, quienes adhiriéndose a la teoría del procesamiento de información, han estructurado el constructo a través de cuatro habilidades:

Percepción, valoración y expresión de las emociones. Hace referencia a la certeza con la que las personas pueden identificar las emociones en ellos mismos y otros. Incluye el registro, la atención y la identificación de los mensajes emocionales, su manifestación se observa en expresiones faciales, movimientos corporales, posturas, tonos de voz etc. Los adolescentes que poseen ésta habilidad pueden leer con exactitud los estados emocionales de las personas a su alrededor al mismo tiempo que reconocen sus propias emociones y son capaces de expresarlas de manera adecuada (Caruso y Salovey, 2004; Mayer y Salovey, 1997).

Facilitación Emocional del Pensamiento. Es la capacidad que se tiene para emplear las emociones como potencializadores de procesos cognitivos. Los adolescentes que han desarrollado la habilidad son pensadores creativos que emplean sus propios estados emocionales para construir nuevas ideas, mejorar su toma de decisiones e inspirar a otros (Caruso y Salovey, 2004; Mayer y Salovey, 1997).

Comprensión Emocional. Se relaciona con el conocimiento cognitivo que tiene el sujeto de sus emociones y cómo dicha información afecta sus procesos de razonamiento. El adolescente que cuenta con ésta habilidad identifica la causa de sus estados emocionales y sus consecuencias; etiqueta correctamente sus emociones; distingue emociones complejas; y hace buenas predicciones sobre lo que la gente podría sentir en situaciones reales (Caruso y Salovey, 2004; Mayer y Salovey, 1997).

Regulación Reflexiva de las Emociones. Es probablemente la habilidad más compleja del modelo de Mayer y Salovey (1997) e implica la capacidad de manejar las propias emociones y las de los demás disminuyendo sus efectos negativos y maximizando los positivos sin reprimirlas o exagerarlas. Los adolescentes que han la habilidad pueden calmarse o permanecer en determinado tipo de humor según la situación lo requiera; animar a otros o ayudarlos a manejar sus estados de ánimos; y estar abiertos a los sentimientos la gente alrededor e inspirarla (Caruso y Salovey, 2004; Mayer y Salovey, 1997).

El modelo antes desarrollado, es el que actualmente cuenta con mayor apoyo empírico entre los investigadores y ha dado origen a diversos instrumentos como el MSCEIT (Mayer,Salovey \& Caruso Emotional Intelligence Trait), el TMMS-48 (Trait Meta Mood Scale with 48 items) y el TMMS-24 (Trait Meta Mood Scale with 24 items) por lo que fue elegido como sustento teórico de éste trabajo (Fernández Berrocal \& Extremera Pacheco, 2005).

\section{METODOLOGÍA}

Por sus características esta investigación se inserta en el paradigma cuantitativo dentro del tipo de estudio correlacional, ya que pretende conocer el grado de asociación entre las variables: estilos parentales e inteligencia emocional. El tipo de diseño empleado fue no experimental y su corte transversal (Hernández Sampieri, Fernández collado \& Baptista Lucio, 2010).

Para alcanzar el objetivo planteado se contó con la participación voluntaria de 196 alumnos de segundo grado de la Escuela Secundaria Técnica Número 96 ubicada al sur de la ciudad de Puebla, las edades de los sujetos oscilan entre 13 y 15 años, de ellos el 54\% de la muestra lo representan 106 hombres y el 46\%, 90 mujeres. A cada estudiante se le explicó las funciones que debe cumplir 
un cuidador en el proceso de crianza y socialización para poder ser considerando como tal, así pues $71 \%$ de la muestra, es decir, 138 alumnos, identifican como sus cuidadores a papá y mamá, en tanto que 36 de ellos, un 18\% de la muestra, sólo a mamá y 6 de ellos, el 3\% de la población total, sólo a papá. Por último, un $8 \%$ de la muestra, compuesta por 16 alumnos, reconocen como sus cuidadores a personas diferentes, ya sean primos, hermanos, abuelos, tíos, etc.

En lo que corresponde a la recolección de datos se emplearon dos instrumentos:

La escala de prácticas parentales para adolescentes de Andrade y Betancourt (2008) surgida de las observaciones de Darling y Steinberg (1993) acerca de la distinción que los autores hacen entre prácticas y estilos de crianza. El instrumento, creado en México, mide la percepción que los adolescentes entre 12 y 18 años tienen acerca de las prácticas de comunicación y control conductual, comprendidas como el conocimiento de las actividades de los hijos, autonomía o el respeto hacia las decisiones de los menores, imposición, es decir, cuánto desean los padres que sus hijos piensen como ellos y control psicológico, o sea la inducción excesiva de sentimientos de culpa y devaluación que los padres emplean en la tarea de educarles. Se compone de 80 reactivos, 40 evalúan al cuidador masculino y 40 al cuidador femenino. Cuenta con un alfa de Cronbach de.907 para ellos y de .840 para ellas, el instrumento ha sido empleado en un amplio número de investigaciones entre las que destacan las de Celis Ochoa, Vallejo Casarín, Osorio Munguía, Rojas Rivera y Reyes García (2011), Palacios Delgado y Andrade Palos (2008), Andrade Palos, Betancourt Ocampo, Vallejo Casarín, Celis Ochoa y Rojas Rivera (2012), Ruvalcaba, Gallegos Caballo y Villegas (2016),

Trait-Meta Mood Scale 24 se basa en la teoría de Mayer y Salovey (1997). La escala mide la inteligencia emocional percibida (IEP) o el metaconocimiento del individuo sobre sus habilidades emocionales en 3 procesos: atención emocional, puntualizada como la capacidad de sentir y expresar Ios sentimientos de forma adecuada; claridad emocional, como la capacidad de comprender correctamente los estados emocionales; y reparación emocional, detallada como la capacidad de regular los estados emocionales correctamente (Fernández-Berrocal, Extremera \& Ramos, 2004). La traducción de éste instrumento al idioma español fue elaborada por Fernández-Berrocal, Extremera y Ramos (2004). En la población mexicana su confiablidad y validez ha sido estudiada por Rodríguez Nieto, Sánchez Miranda, Valdivia Vázquez y Padilla Montemayor (2005) y Valdivia Vázquez, Rubio Sosa y French (2014).

La aplicación de estos instrumentos se realizó en un solo día luego de que las autoridades de la institución la aprobaran, previa exposición de los fines de investigación y organizar tiempos con el tutor responsable de los cinco grupos de segundo grado a fin de no afectar las actividades.

Todos los alumnos participaron de manera voluntaria y se les informó sobre la confidencialidad de sus respuestas. También se les explicó la intención de las escalas a contestar y la manera de hacerlo.

Posteriormente se elaboró el registro, procesamiento y análisis descriptivo de los datos en el programa estadístico SPSS.V21

\section{RESULTADOS}

Enseguida se muestran los hallazgos obtenidos del análisis de los datos. En primer lugar, se describen los referentes a prácticas parentales, posteriormente, Ios relacionados con IE para finalizar abordando la correlación entre ambas variables.

\section{Prácticas empleadas por cuidadores masculinos y femeninos}

Tomando en consideración que la escala de prácticas parentales de Andrade y Betancourt (2008) se integra por un instrumento que evalúa a cuidadores femeninos y masculinos se presentan a continuación los resultados en función del sexo del cuidador. 


\section{PRÁCTICAS PARENTALES E INTELIGENCIA EMOCIONAL EN ESTUDIANTES DE SECUNDARIA. UN ESTUDIO CORRELACIONAL}

Lo primero a resaltar para el caso de los cuidadores del género masculino es que de 196 sujetos participantes 18 de ellos no identifican una figura masculina a cargo de su crianza, por lo tanto, los siguientes números equivalen a los 178 padres. Así pues, en 100 de los casos, que representan al $56 \%$ de la muestra, las prácticas más empleadas son las de concesión de autonomía; le siguen prácticas de comunicación y control conductual percibidas por un 34\% de la población, es decir, en 64 casos, como las más comunes. El 8\% de los alumnos, 14 de ellos, manifiestan que las prácticas mayormente empleadas por su cuidador masculino son las de imposición; mientras que en último lugar aparecen las prácticas de control psicológico, reportadas por 4 sujetos quienes representan el $2 \%$ de la población. Estos valores pueden apreciarse en la Gráfica 1.

Gráfica 1. Percepción de las prácticas empleadas por los cuidadores del género masculino

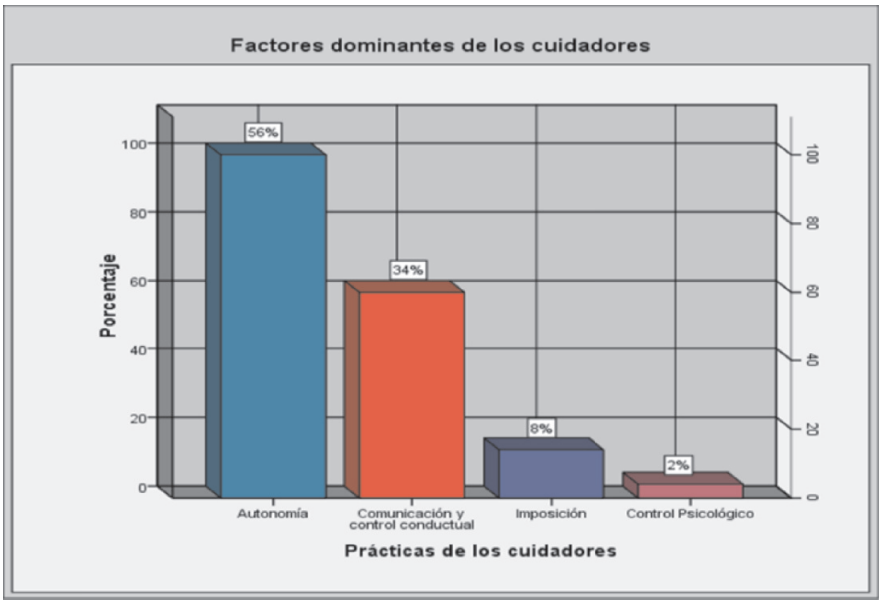

En cuanto a las cuidadoras, la Gráfica 2 muestra la percepción de las prácticas que emplean los cuidadores del género femenino en la ejecución de sus tareas de crianza. En este caso sólo tres sujetos no identifican una figura femenina involucrada en su crianza, por lo tanto, los siguientes números reflejan los resultados obtenidos por 193 cuidadoras. De este modo, la práctica percibida como la más utilizada es el control conductual identificada por 86 alumnos, es decir, el $45 \%$ de la población, seguida de las de comunicación registradas en 60 casos que representan el $31 \%$ de la muestra. En lo que respecta a las prácticas de concesión de autonomía su uso fue percibido por un 16\% de los alumnos, es decir 31 adolescentes. En menor porcentaje se reportan prácticas de imposición detectadas en el $6 \%$, integrado por 12 alumnos. En cuanto a las prácticas de control psicológico son percibidas como las menos recurrentes por un $2 \%$ de la muestra que representa cuatro de los casos. 
Gráfica 2. Percepción de las prácticas empleadas por los cuidadores del género femenino

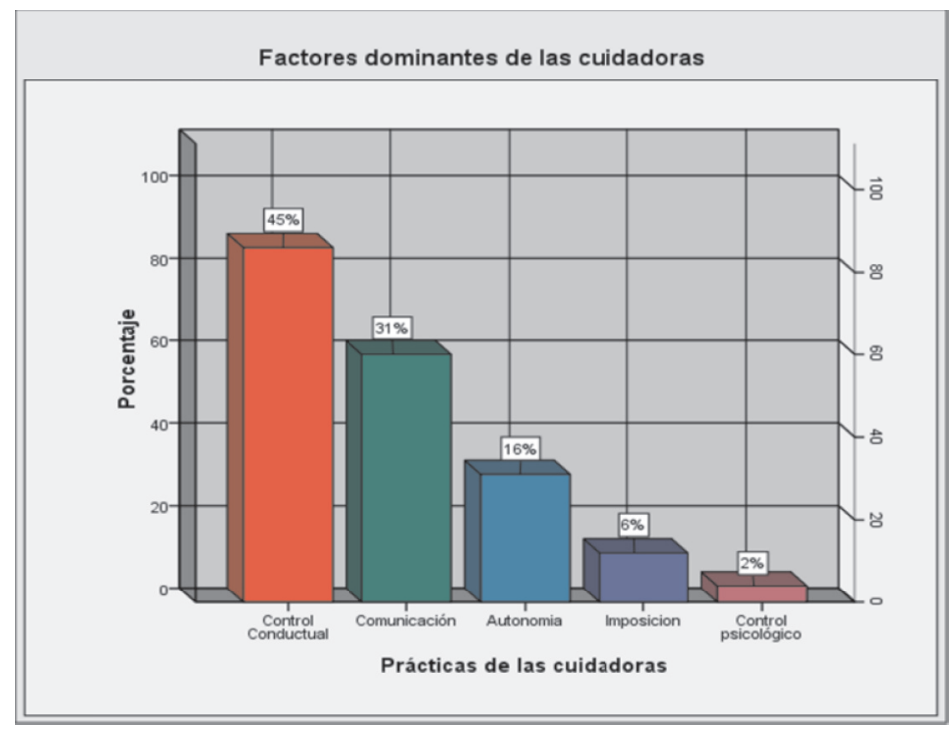

Puede observarse que estos resultados no presentan grandes diferencias entre cuidadores y cuidadoras. Las prácticas parentales de control conductual, comunicación y autonomía son las más percibidas por los alumnos como dominantes entre los adolescentes.

\section{Inteligencia Emocional}

En la Tabla 1, se muestran los resultados obtenidos a través del análisis del TMMS-24 donde se puede apreciar que en relación a la Atención Emocional en 91 casos, mismos que representan el $46 \%$ de la muestra, los adolescentes deben mejorar la habilidad; en 24 casos, es decir el 13\%, presenta niveles muy altos, por lo que igual manera deben mejorar la habilidad; y en 81 casos, que corresponden al $41 \%$ de la muestra cuentan con niveles adecuados de la habilidad.

Con respecto a la Claridad Emocional se aprecia que un $48 \%$ de la muestra, 96 alumnos, deben mejorar su claridad; en tanto que el 39\%, 76 estudiantes, presentan niveles adecuados de la habilidad; y el $13 \%$ cuenta con excelentes niveles de claridad emocional.

En cuanto a la Reparación Emocional se encuentra que la habilidad debe ser mejorada en 68 alumnos, quienes representan el $35 \%$ de la muestra; mientras que 89 de ellos, es decir el $45 \%$, muestra niveles adecuados; y sólo un $20 \%$, que equivale a 39 participantes, perciben poseer niveles excelentes de regulación emocional. 
PRÁCTICAS PARENTALES E INTELIGENCIA EMOCIONAL EN ESTUDIANTES DE SECUNDARIA.

UN ESTUDIO CORRELACIONAL

Tabla I. Inteligencia Emocional en Estudiante de Secundaria

\begin{tabular}{|c|c|c|c|c|c|}
\hline \multicolumn{6}{|c|}{ Habilidades emocionales presentes en los alumnos } \\
\hline \multirow[t]{2}{*}{ Habilidades } & & \multicolumn{3}{|c|}{ Nivel de habilidad } & \multirow[t]{2}{*}{ Totales } \\
\hline & & Debe mejorar & Adecuado & $\begin{array}{c}\text { Debe } \\
\text { mejorar }\end{array}$ & \\
\hline \multirow{3}{*}{ Atención emocional } & Frecuencia & 91 & 81 & $24 *$ & 196 \\
\hline & Porcentaje & $46 \%$ & $41 \%$ & $13 \%$ & $100 \%$ \\
\hline & & Debe mejorar & Adecuado & Excelente & \\
\hline \multirow{3}{*}{ Claridad emocional } & Frecuencia & 95 & 76 & 25 & 196 \\
\hline & Porcentaje & $48 \%$ & $39 \%$ & $13 \%$ & $100 \%$ \\
\hline & & Debe mejorar & Adecuado & Excelente & \\
\hline \multirow{2}{*}{ Reparación emocional } & Frecuencia & 68 & 89 & 39 & 196 \\
\hline & Porcentaje & $35 \%$ & $45 \%$ & $20 \%$ & $100 \%$ \\
\hline
\end{tabular}

\section{Correlaciones entre Prácticas Parentales e Inteligencia Emocional}

En la Tabla 2 se muestran las correlaciones existentes entre las prácticas parentales de los cuidadores masculinos y las habilidades de la IE de los sujetos. De esta forma se aprecia una correlación positiva débil entre la práctica parental comunicación y control conductual y las habilidades: Atención Emocional $(r=.315 ; p<.01)$ y Claridad Emocional $(r=.375 ; p<.01)$. También fue detectada una correlación positiva de mediana intensidad con la habilidad reparación emocional $(r=.509$; $p<.01)$.

Igualmente existen correlaciones positivas débiles entre las prácticas de autonomía y las habilidades Atención Emocional $(r=.254, p<.01)$, Claridad Emocional $(r=.255, p<.01)$ y Reparación Emocional ( $r=.377, p<.01)$. En el caso de las prácticas de imposición y control psicológico no se hallaron correlaciones con ninguna habilidad de la IE.

Tabla II. Prácticas de los cuidadores del género masculino y habilidades emocionales de los adolescentes

\begin{tabular}{cccc}
\hline \multicolumn{4}{c}{ Habilidades de la IE } \\
\hline & $\begin{array}{c}\text { Atención } \\
\text { emocional }\end{array}$ & $\begin{array}{c}\text { Claridad } \\
\text { emocional }\end{array}$ & $\begin{array}{c}\text { Reparación } \\
\text { emocional }\end{array}$ \\
\hline & \multicolumn{2}{c}{ Dimensiones de los cuidadores } \\
\hline $\begin{array}{c}\text { Comunicación y } \\
\text { control conductual }\end{array}$ & $.315^{* *}$ & $.375^{* *}$ & $.509^{* *}$ \\
$\quad$ Autonomía & $.254^{* *}$ & $.255^{* *}$ & $.377^{* *}$ \\
$\quad \begin{array}{l}\text { Imposición } \\
\text { Control psicológico }\end{array}$ & .039 & .093 & .029 \\
\hline$*$ p $<.01$ & .048 & -.134 & -.104 \\
\hline
\end{tabular}

En el caso de las prácticas parentales de los cuidadores femeninos y las habilidades de la IE de los sujetos en la Tabla 3 se muestra que existe una correlación positiva débil entre la práctica comunicación y las habilidades: Atención emocional $(r=.230, p<.01)$, Claridad Emocional $(r=.408, p<.01)$ y Reparación Emocional $(r=.433, p<.01)$. También se observan correlaciones positivas débiles entre 
las prácticas de autonomía y las habilidades Atención emocional $(r=.202, p<.01)$, Claridad Emocional $(r=.183 p<.05)$ y Reparación Emocional $(r=.262, p<.01)$. En el caso de las prácticas de control psicológico las correlaciones son igualmente débiles, pero en sentido negativo, no obstante, ocurren sólo con las habilidades de Claridad Emocional $(r=-.149, p<.05)$ y Reparación Emocional $(r=-.189, p<.01)$. Finalmente, se advierten correlaciones positivas débiles entre las prácticas de control conductual y las habilidades Atención Emocional $(r=.291, p<.01)$, Claridad Emocional $(r=.360, p<.01)$ y Reparación Emocional $(r=.446, p<.01)$. Sólo en el caso de las prácticas de imposición no existen correlaciones.

Tabla III. Prácticas de las cuidadoras del género femenino y habilidades emocionales de los adolescentes

\begin{tabular}{cccc}
\hline \multicolumn{4}{c}{ Habilidades de la IE } \\
\hline & $\begin{array}{c}\text { Atención } \\
\text { emocional }\end{array}$ & $\begin{array}{c}\text { Claridad } \\
\text { emocional }\end{array}$ & $\begin{array}{c}\text { Reparación } \\
\text { emocional }\end{array}$ \\
\hline & Dimensiones de las cuidadoras \\
\hline Comunicación & $.230^{* *}$ & $.408^{* *}$ & $.433^{* *}$ \\
Autonomía & $.202^{* *}$ & $.183^{*}$ & $.262^{* *}$ \\
Imposición & .025 & .133 & .044 \\
Control psicológico & -.126 & $-.149^{*}$ & $-.189^{* *}$ \\
Control conductual & $.291^{* *}$ & $.360^{* *}$ & $.446^{* *}$ \\
\hline
\end{tabular}

$* \mathrm{p}<.05, * * \mathrm{p}<.01$

\section{CONCLUSIONES Y DISCUSIÓN}

Como se puede apreciar dentro del apartado de resultados, en los cuidadores se encontró una correlación positiva de débil a media entre las prácticas comunicación; control conductual; autonomía y las habilidades atención, claridad y reparación emocional.

Resultados similares se hallaron en las cuidadoras a la par que se encontró una correlación negativa débil entre control psicológico y las habilidades atención, claridad y reparación emocional.

Por lo anterior se rechaza la hipótesis nula y se acepta la de investigación que afirma la existencia de correlaciones entre las prácticas parentales y la inteligencia emocional

Esto respalda las afirmaciones de Andrade y Betancourt (2010) quienes suponen que la prácticas autonomía, comunicación y control conductual indican una adecuada interacción entre padres e hijos lo cual tendría efectos positivos sobre la IE en alumnos de secundaria. Su ausencia o escasa presencia se asocia con una mayor probabilidad padecer diversas alteraciones como la depresión.

En cuanto a las correlaciones negativas detectadas entre la práctica del control psicológico y las habilidades de la inteligencia emocional, autores como Meier y Oros (2012) afirman que las prácticas parentales negativas, como la autonomía extrema y control patológico, ejercen un efecto adverso sobre las emociones ya que la relación con los padres determina un perfil emocional. La presencia de control psicológico es asociada con mayor crítica y rechazo de los padres hacia los hijos (Bathia, 20012; Capano Bosch et al., 2016)

Así pues, las prácticas parentales son una poderosa herramienta para el desarrollo de la IE en Ios adolescentes, sin embargo, hay que tener en cuenta que los padres son solo uno de los actores que pueden ayudar al desarrollo socioafectivo de los jóvenes, como muestra la intensidad de las 


\section{PRÁCTICAS PARENTALES E INTELIGENCIA EMOCIONAL EN ESTUDIANTES DE SECUNDARIA. UN ESTUDIO CORRELACIONAL}

correlaciones encontradas en el estudio. Y si bien existe una relación entre las prácticas parentales y la IE los docentes también deben incluir implementar dentro del aula actividades que refuercen la educación emocional a través de técnicas vivenciales que ayuden a los adolescentes a la resolución de problemas dentro y fuera del ámbito escolar de modo que se contemple a las personas en términos más integrales al fomentar el desarrollo de su autonomía, autoconfianza y se les faculte para tomar decisiones y responsabilizarse por los resultados de estas, esto supone que la escuela debe verse no sólo como un escenario de desarrollo cognitivo sino de habilidades para la vida y que, no obstante, para que los alumnos sean capaces de aprehenderlas precisan que tanto la familia como las instituciones escolares trabajen en conjunto, aún más, se precisa que los trabajos que pretenden impulsar mejores en esta área tengan una visión extendida en el tiempo de modo que no se abandonen como ha ocurrido en muchos casos y que, a pesar de las investigaciones ya realizadas, estas profundicen tomando como punto de referencia las necesidades señaladas por los propios adolescentes pues de otro modo es probable que los esfuerzos no tengan sentido para ellos y es hacia allá donde deberían fijarse las miradas (Dueñas Buey, 2002; Bello Dávila, Rionda Sánchez \& Rodríguez Pérez, 2010; Fernández Berrocal y Extremera Pacheco, 2005).

\section{REFERENCIAS BIBLIOGRÁFICAS}

Andrade Palos, P., \& Betancourt Ocampo, D. (2008). Manual de la Escala de Prácticas Parentales Percibidas. México: Universidad Nacional Autónoma de México.

Andrade Palos, P., Betancourt Ocampo, D., Vallejo Casarin, A., Celis Ochoa, B., \& Rojas Rivera, R. M. (2012). Prácticas parentales y sintomatología depresiva en adolescents. Salud Mental, 35(1), 29-36. Recuperado de: http://www.scielo.org.mx/pdf/sm/v35n1/v35n1a5.pdf

Aroca Montolío, C. y Cánovas Leonhardt, P. (2012). Los estilos educativos parentales desde los modelos interactivo y de construcción conjunta: Revisión de las investigaciones. Teoría de la educación. Revista interuniversitaria, 24, (2), 149 - 176. Recuperado de: http://revistas.usal.es/index.php/1130-3743/article/view/10359/10798

Bar-On, R. (2006). The Bar-On model of emotional- social intelligence (ESI). Psicothema, 18, (1), 13-25. Recuperado de http://www.psicothema.com/psicothema.asp?id=3271

Barudy, J. y Dantagnan, M. (2005). Los buenos tratos a la infancia: Parentalidad, apego y resiliencia. España: Gedisa.

Bathia, G. (2012). A study of family relationship in relation to emotional intelligence of the students of secondary level. International Journal of Scientific and Research Publications, 2(12), 1-5. Recuperado de http://www.ijsrp.org/research-paper-1212/ijsrp-p1210.pdf

Baumrind, D. (1967). Child care practices anteceding three patterns of preschool behavior, Genetic psychology monographs, 75, (1), 43, 162 - 173. Recuperado de: http://homepages.utoledo.edu /mcaruso/honors-lifespan/baumrind.PDF

Baumrind, D. (1966). Effects of authoritative parental control on child behavior. Child development, 37(4), 887-907. Recuperado de http://persweb.wabash.edu/facstaff/hortonr/ articles\%20for\%20class/baumrind.pdf

Bello-Dávila, Z., Rionda-Sánchez, H. D. y Rodríguez Pérez, M. E. (2010). La inteligencia emocional y su educación. VARONA, (51), 36 - 43. Recuperado de http://www.redalyc.org/ pdf/3606/360635569006.pdf

Bilimler Dergisi, S. (2006). Emotional intelligence and family environment. SAYI, 8, (16), 169-175. Recuperado de http://journals.manas.edu.kg/mjsr/oldarchives/Vol08_Issue16_2006/537-14251-PB.pdf

Bisquerra Alzina, R. (2007). Educación emocional y Bienestar. Barcelona: Praxis

Bisquerra Alzina, R. (2009). Psicopedagogía de las Emociones. España: Síntesis 
Capano Bosch, A. Del Luján González Tornaría, M., \& Massonier, N. (2016). Estilos relacionales parentales: Estudio con adolescentes y sus padres. Revista de Psicología, 34, (2), 413-444. Recuperado de http://www.redalyc.org/pdf/3378/337846349009.pdf

Capano, A. y Ubach, A. (2013). Estilos parentales, parentalidad positiva y formación de padres. Ciencias psicológicas, 7, (1), 83 - 95. Recuperado de: http://www.scielo.edu.uy /pdf/cp/v7n1/v7n1a08.pdf

Celis Ochoa, H. B., Vallejo Casarín, A. G., Osorno Munguía, J. R., Rojas Rivera, R. M., \& Reyes García, S. I. (2011). La escala de prácticas parentales de Andrade y Betancourt en adolescentes veracruzanos. Revista de educación y desarrollo, (18), 67-73. Recuperado de http://www.cucs.udg.mx/revistas/edu_desarrollo/anteriores/18/018_Segura.pdf

Cobos Sánchez, L., Flujas Contreras, J. M., \& Gómez Becerra, I. (2017). The role of emotional intelligence in psychological adjustment among adolescents. Anales de Psicología, 33(1), 66-73. Recuperado de: http://www.redalyc.org/pdf/167/16749090009.pdf

Cooper R., \& Sawaf, A. (1996). La Inteligencia Emocional Aplicada al Liderazgo y a las Organizaciones. Colombia: Norma

Cuervo Martínez, A. (2010). Pautas de crianza y desarrollo socioafectivo en la infancia. Diversitas: Perspectivas en Psicología, 6(1), 111-121. Recuperado de http://www.redalyc.org/ pdf/679/67916261009.pdf

Darling, N., \& Steinberg, L. (1993). Parenting style as context: An integrative model. Psychological Bulletin, 113(3), 487-496. Recuperado de: http://www2.oberlin.edu/faculty/ndarling/lab/psychbull.pdf

Dueñas Buey, M. L. (2002). Importancia de la inteligencia emocional: un nuevo reto para la orientación educativa. Educación XX1, (5), 77 - 96. Recuperado de http://www.redalyc.org/ articulo.0a?id=70600505

Espinal, I., Gimeno, A., \& González, F. (2004). El enfoque sistémico en los estudios sobre la familia. Revista internacional de sistemas, (14), 21-34. Recuperado de: https://www.uv.es/jugar2/Enfoque\%20Sistemico.pdf

Fernández-Berrocal, P., Extremera, N., \& Ramos, N. (2004). Validity and reability of the spanish modified version of Trait Meta Mood Scale. Psychological Reports, (94), 751-755. Recuperado de http://emotional.intelligence.uma.es/pdfs/Spanish\%20TMMS.pdf

Fernández Berrocal, P. y Extremera Pacheco, N. (2005). La inteligencia emocional y la educación de las emociones desde el modelo de Mayer y Salovey. Revista interuniversitaria de formación del profesorado, 19, (3), 63 - 93. Recuperado de http://emotional.intelligence.uma.es/documentos/pdf61modelo_de_mayer_salovey.pdf

Fernández Berrocal, P., \& Ruiz Aranda, D. (2008). La inteligencia emocional en la educación. Revista Electrónica de Investigación Psicoeducativa, 6(2), 421- 426. Recuperado de: http://www.investigacion-psicopedagogica.org/revista/articulos/15/espannol/Art_15_256.pdf

Ferragut, M., \& Fierro, A. (2012). Inteligencia emocional, bienestar personal y rendimiento académico en preadolescentes. Revista Latinoamericana de Psicología, 44(3), 95-104. Recuperado de: http://www.redalyc.org/articulo.oa?id=80525022008

Frías Armenta, M., Fraijo Sing, B., \& Tapia Fonllem, C. (2012). Prácticas de crianza y desarrollo afectivo y emocional de los niños. PSICUMEX, 4(2), 30 - 41. Recuperado de http://www.psicumex.uson.mx/revistas/articulos/4-r4_art3.pdf

Gil-Olarte Márquez, P., Guil Bozal, R., Serrano Díaz, N., \& Larrán Escandón,C. (2014). Inteligencia emocional y clima familiar. International Journal of Developmental and Educational Psychology, 2(1), 407 - 417. Recuperado de: http://www.redalyc.org/pdf/3498/349851782043.pdf 


\section{PRÁCTICAS PARENTALES E INTELIGENCIA EMOCIONAL EN ESTUDIANTES DE SECUNDARIA. UN ESTUDIO CORRELACIONAL}

Goleman, D. (2000). La Inteligencia Emocional Aplicada a las Organizaciones. Barcelona: Kairos Goleman, D. (2002). La Inteligencia Emocional. México: Vergara.

Hernández Sampieri, R., Fernández Collado, C. y Baptista Lucio, M. P. (2010). Metodología de la investigación. México: McGraw-Hill.

Malander, N. (2016). Percepción de prácticas parentales y estrategias de aprendizaje en estudiantes secundarios. Revista de Psicología, 25(1), 1-19. Recuperado de: http://www.redalyc.org/ pdf/264/26446630004.pdf

Mayer, J. D., \& Salovey, P. (1997). Emotional development and emotional intelligence. New York: BasicBooks.

Meier, L. K., \& Oros, L. B. (2012). Percepción de las prácticas parentales y experiencia de emociones positivas en adolescentes. Revista de psicología, 8 (16), 73-84. Recuperado de: http://ri.conicet.gov.ar/bitstream/handle/11336/19108/selection\%20\%283\%29.pdf?sequen$\mathrm{ce}=2 \&$ isAllowed $=\mathrm{y}$

Méndez Sánchez, M. P., Andrade Palos, P., \& Peñaloza Gómez, R. (2013). Prácticas parentales y capacidades y dificultades en preadolescentes. Revista Intercontinental de Psicología y Educación, 15, (1), 99 - 118. Recuperado de: http://www.redalyc.org/pdf/802/80225697007.pdf

Mestre Navas, J.M., Guil Bozal, R., \& Mestre Romero, R. (2005). Inteligencia emocional: Resultados preliminares sobre su naturaleza a partir de un estudio correlacional en muestras de estudiantes de secundaria. Revista Española de Orientación y Psicopedagogía, 16, (2), 269-281. Recuperado de: http://www.redalyc.org/articulo.oa?id=338230773005

Nardone, G., Giannotti, E. y Rocchi, R. (2003). Modelos de familia. España: Herder.

Palacios Delgado, J. R., \& Andrade Palos, P. (2008). Influencia de las prácticas parentales en las conductas problema en adolescentes. Investigación universitaria multidisciplinaria, (7), 7-18. Recuperado de: https://dialnet.unirioja.es/descarga/articulo/2986557.pdf

Papalia, D. E., Wendkos Olds, S., \& Duskin Feldman, R. (2009). Psicología del desarrollo. De la infancia a la adolescencia. México: Mc Graw Hill.

Ramírez, M. A. (2005). Padres y desarrollo de los hijos: Prácticas de crianza. Estudios pedagógicos, 31(2), 167-177. Recuperado de: http://www.redalyc.org/articulo.oa?id=173519073011

Rodrigo López, M. J., \& Palacios, J. (1998). Familia y desarrollo humano. España: Alianza.

Rodríguez Nieto, M.A., Sánchez Miranda, M. P., Valdivia Vázquez, J.A., \& Padilla Montemayor, V. M. (2005). Perfil de inteligencia emocional en estudiantes universitarios de la facultad de psicología. Universidad Autónoma de Nuevo León. Recuperado de http://www.uas/p.mx/PDF/ 2228_320.pdf

Ruvalcaba, N., Gallegos, J., Caballo, V. \& Villegas, D. (2016). Prácticas parentales e indicadores de salud mental en adolescentes. Psicología desde el caribe, 33(3), 223-236. Recuperado de: https://www.researchgate.net/publication/314182709

Salovey, P., \& Mayer, J. (1990). Emotional intelligence. Imagination, Cognition, and Personality, 9(3), 185-211.

Valdivia Vázquez, J.A., Rubio Sosa, J.C., \& French, B.F. (2014). Examination of the Spanish Trait Meta-Mood Scale-24 Factor Structure in a Mexican Setting. Journal of Psychoeducational Assessment, 33(5), 473-482. doi: 10.1177/0734282914552052

Vázquez Rúa, C. I. (2005). Las nuevas tipologías familiares y los malestares interrelacionales que se suscitan en ellas. Revista Virtual Universidad Católica del Norte, (14). Recuperado de: http://www.redalyc.org/articulo.oa?id=194220381002 